\title{
Specific expression of miR-17-5p and miR-127 in testicular and central nervous system diffuse large B-cell lymphoma
}

\author{
Jan-Lukas Robertus ${ }^{1}$, Geert Harms ${ }^{1}$, Tjasso Blokzijl ${ }^{1}$, Marije Booman ${ }^{1}$, Daphne de Jong ${ }^{3}$, \\ Gustaaf van Imhoff ${ }^{2}$, Stefano Rosati ${ }^{1}$, Ed Schuuring ${ }^{1}$, Philip Kluin ${ }^{1}$ and Anke van den Berg ${ }^{1}$ \\ ${ }^{1}$ Department of Pathology and Laboratory Medicine, University Medical Center Groningen, University of \\ Groningen, Groningen, The Netherlands; ${ }^{2}$ Department of Hematology, University Medical Center Groningen, \\ University of Groningen, Groningen, The Netherlands and ${ }^{3}$ Department of Pathology, The Netherlands \\ Cancer Institute, Amsterdam, The Netherlands
}

\begin{abstract}
Recent studies have shown that certain non-coding short RNAs, called miRNAs, play an important role in diffuse large B-cell lymphomas. Patients with diffuse large B-cell lymphoma have great diversity in both clinical characteristics, site of presentation and outcome. The aim of our study is to validate the differential expression in germinal center and non-germinal center diffuse large B-cell lymphoma,s and to study to the extent to which the primary site of differentiation is associated with the miRNA expression profile. We studied 50 cases of de novo diffuse large B-cell lymphoma for the expression of 15 miRNAs (miR-15a, miR-15b, miR-16, miR-17-3p, miR-17-5p, miR-18a, miR-19a, miR-19b, miR-20a, miR-21, miR-92, miR-127, miR-155, miR-181a and miR-221). Apart from 19 nodal cases without extranodal dissemination (stages I and II), we selected two groups with unambiguous stages I and II extranodal presentation; 9 cases of primary central nervous system, 11 cases of primary testicular and 11 cases of other primary extranodal diffuse large B-cell lymphomas. All cases were analyzed with qRT-PCR. In situ hybridization for the most differentially expressed miRNAs was performed to show miRNA expression in tumor cells, but not in background cells. MiR-21 and miR-19b showed the highest expression levels. No significant differences were seen between germinal center and non-germinal center diffuse large B-cell lymphomas in either the total or the nodal group for any of the 15 miRNAs. Two miRNAs showed significant differences in expression levels for diffuse large B-cell lymphoma subgroups according to the site of presentation. MiR-17-5p showed a significant higher expression level in the central nervous system compared with testicular and nodal diffuse large B-cell lymphomas $(P<0.05)$. MiR-127 levels were significantly higher in testicular than in central nervous system and in nodal diffuse large B-cell lymphomas $(P<0.05)$. We conclude that the location of diffuse large B-cell lymphoma is an important factor in determining the differential expression of miRNAs.
\end{abstract}

Modern Pathology (2009) 22, 547-555; doi:10.1038/modpathol.2009.10; published online 13 March 2009

Keywords: diffuse large B-cell lymphoma; miRNA; CNS; testicular

Diffuse large B-cell lymphoma (BCL) accounts for approximately $40 \%$ of all adult non-Hodgkin lymphomas. Diffuse large BCL is a clinically heterogeneous group of lymphomas, which presents at different anatomical sites, either nodal or extranodal. Anatomical sites, such as the testis and central nervous system, show marked differences in genetic aberrations, morphology and clinical behavior. ${ }^{1,2}$ Testicular diffuse large BCL and central nervous

Correspondence: Dr J-L Robertus, Department of Pathology and Laboratory Medicine, University Medical Center Groningen, Hanzeplein 1, 4700 RB Groningen, The Netherlands.

E-mail: j.l.robertus@path.umcg.nl

Received 6 June 2008; revised 13 October 2008; accepted 19 October 2008; published online 13 March 2009 system diffuse large BCL both have a different biological and clinical behavior compared with other nodal and extranodal diffuse large BCLs.

Gene expression profiling of untreated de novo diffuse large BCL has revealed three distinct subgroups. The first with the characteristics of normal germinal center B cells, the second with the characteristics of activated blood memory $\mathrm{B}$ cells and a third group to which no profile could be assigned. The germinal center subgroup has a significantly better prognosis than the other two non-germinal center subgroups. ${ }^{3-5}$ The introduction of rituximab to the standard treatment of patients with diffuse large BCL revealed an increase in complete-response rate and overall survival. ${ }^{6}$ More recent studies indicate that the addition of rituximab has improved 
the progression-free and overall survival rate, specifically for activated B-cell type diffuse large BCL, ${ }^{7,8}$ thus blurring the prognostic differences between diffuse large BCL of germinal center and of activated $\mathrm{B}$-cell origin.

Recently, a group of 21-23 nucleotide long microRNAs (miRNAs) was discovered. These miRNAs post-transcriptionally downregulate protein expression on the basis of limited sequence complementarity to the $3^{\prime}$-UTR of mRNA transcripts. ${ }^{9}$ Many of these miRNAs are involved in cancer by targeting oncogenes or tumor suppressor genes. ${ }^{10-13}$ Certain miRNAs are reported to play a role in B-cell malignancies and in B-cell development;for example, miR-181a overexpression induces a higher percentage B-lineage cells in both tissue culture assays and adult mice cells. ${ }^{12,14-17}$

One of the first miRNAs linked to diffuse large BCL was miR-155, which is derived from a longer pri-miR-155, also referred to as BIC. Both BIC and miR-155 have a higher expression level in activated diffuse large BCL when compared with that in germinal center diffuse large BCL. ${ }^{14,18-21}$ A putative oncogenic role for this miRNA was supported by the spontaneous development of a polyclonal preleukemic B-cell proliferation in an E $\mu$-miR-155 transgenic mice model. ${ }^{22}$ Two other miRNAs, miR-21 and 221, have also been reported to be expressed at higher levels in non-germinal center diffuse large BCL cell lines and cases. ${ }^{21}$ Both of these miRNAs target the antiapoptotic BCL2 gene. MiR-15a and miR-16-1 also target BCL2, and miR-16-1 downregulates cell growth and cell cycle progression. ${ }^{19}$ These two miRNAs are the most likely targets of the characteristic 13q14 deletion in CLL, and are downregulated in $75 \%$ of CLL cases. ${ }^{14,19,20}$ BCL6 is a target of miR127 , and this gene has been reported to be downregulated after demethylation of the promoter region and concurrent higher expression of miR-127. In diffuse large BCL, amplification of $13 \mathrm{q} 31$ is associated with the overexpression of the pri-miRNA transcript, C13ORF25, containing seven miRNAs, miR-18a, miR-19a, miR-19b, miR-20a, miR-17-3p, miR-17-5p and miR-92. ${ }^{23-25}$

Our goal was to determine whether possible differences in expression levels of miRNAs are related to the differences between germinal and non-germinal center diffuse large BCLs, or to the primary site of presentation and biological origin of these lymphomas. To that end, we selected two groups of distinct extranodal diffuse large BCL cases localized in the testis and central nervous system, and compared these groups with other primary extranodal and nodal lymphomas.

\section{Materials and methods}

\section{Patients and Materials}

Forty-one cases of primary Ann Arbor stage I and II nodal and extranodal diffuse large BCLs were obtained from the tissue bank at the Department of Pathology and Medical Biology, University Medical Center Groningen, Groningen and 10 cases from the Department of Pathology, The Netherlands Cancer Institute, Amsterdam. Each case was reviewed by a pathologist and a hematologist. Formalin-fixed paraffin-embedded tissue sections were stained with hematoxylin and eosin (HE) to determine the percentage of tumor cells. Blocks with a tumor cell percentage of $80 \%$ or more were used for RNA isolation. All protocols for obtaining and studying human tissues and cells were approved by the institution's review board for human subject research.

\section{Tissue Microarray (TMA)}

For immunohistochemistry, a TMA was generated with three $0.6-\mathrm{mm}$ cores per case inserted in a grid pattern into a recipient paraffin block using an MT1 manual tissue arrayer (Beecher Instruments, Sun Prairie, USA). Sections were cut $(5 \mu \mathrm{m})$ and immunohistochemical staining was performed with mouse antihuman monoclonal antibodies against CD10 (1:20, 56C6, Novacastra, Newcastle, UK), MUM1 (1:25, MUM1p, DAKO, Copenhagen, Denmark) and BCL6 (1:20, PG-BP, DAKO) after antigen retrieval. Positive staining was visualized using a peroxidase-labeled second step and staining with diaminobenzidine (DAB). Diffuse large BCL cases were classified as germinal center B-cell-like (germinal center) or non-germinal center B-cell-like (non-germinal center) according to the algorithm as described by Hans et al. ${ }^{3}$ A uniform cutoff level of $30 \%$ was chosen for CD10, BCL6 and MUM1.

\section{RNA In Situ Hybridiztion (RNA ISH)}

RNA in situ hybridization was performed as described earlier. ${ }^{18,26}$ miRNA staining was performed using an LNA probe antisense to miR-17-5p and miR-127-3p (Exiqon, Denmark) in three central nervous system diffuse large BCL cases and in three testicular diffuse large BCL cases, according to the manufacturer's protocol.

\section{RNA Isolation}

RNA isolation was performed using the protocol as described by Specht et al. ${ }^{27}$ DNAse treatment was performed using a Turbo DNA free kit (Ambion, Woodward, USA) according to the manufacturer's instructions. Efficiency of the DNase procedure was checked using a multiplex PCR with 5 primer sets specific for different genomic DNA loci, and subsequent analysis on a $1.5 \%$ agarose gel. No PCR products were seen confirming the effectiveness of the DNAse treatment. RNA concentrations were determined on a NanoDrop ${ }^{\circledR}$ ND-1000 Spectrophotometer (Nano Drop Technologies, Wilmington, Delaware, USA). 
qRT-PCR of Mature miRNAs and Primary miRNA Transcripts

MiRNA reverse transcription of $5 \mathrm{ng}$ total RNA was performed using a Taqman microRNA reverse transcription kit and miRNA-specific primers for miR15a, miR-15b, miR-16, miR-17-3p, miR-17-5p, miR18a, miR-19a, miR-19b, miR-20a, miR -21, miR-92, miR-127, miR-155, miR-181a and miR-221 in accordance with the instructions supplied by the manufacturer (Applied Biosystems, Foster City, USA). Superscript II (Invitrogen, Carlsbad, USA) and random hexamer primers using $200 \mathrm{ng}$ of total RNA were used for cDNA synthesis. The subsequent quantitative amplification of $U 6$ was performed using Taqman microRNA and Taqman gene expression assays, together with the 7900HT ABI fast real-time PCR system (Applied Biosystems). All procedures were in accordance with the instructions of the manufacturers. PCR reactions were performed in triplicate; positive and negative controls were included in each run with an input of $0.44 \mathrm{ng}$ for miRNAs and of $2 \mathrm{ng}$ for the other genes. Fluorescence was quantified with the sequence detection system software SDS (version 2.1, Applied Biosystems). Mean cycle threshold $\left(C_{t}\right)$ values and standard deviations were calculated for all miRNAs and genes. U6 was selected from several other housekeeping genes (GAPDH, RP2, HPRT, TBP and $B 2 M$ ), as it showed a uniform expression level in all samples and a sufficiently low $C_{\mathrm{t}}$ value (median: 22; s.d.: 1.7). Samples with a $C_{\mathrm{t}}$ value for $U 6>30$ were excluded from further analysis. The amount of target miRNA was normalized relative to the amount of $U 6\left(\Delta C_{\mathrm{t}}=\Delta C_{\mathrm{tmiRNA}}-\Delta C_{\mathrm{tU}}\right)$. Relative expression levels were expressed as $2^{-\Delta C_{\mathrm{t}}}$ (Supplementary Table 1).

\section{Data Analysis}

To determine significant differences between testicular, central nervous system, nodal and other extranodal diffuse large BCLs, a Kruskal-Wallis test was performed with Dunn's multiple comparison test, and a $P$-value $<0.05$ was considered significant (GraphPad Prism software, version 3.02).

To determine significant differences between germinal and non-germinal center diffuse large BCLs for a specific miRNA, a two-tailed MannWhitney $U$-test was used, and a $P$-value $<0.05$ was considered significant (SPSS, version 14).

Univariate analysis was performed with a Pearson's $\chi^{2}$-test for gender, stage and localization (SPSS, version 14), and a Student's t-test was used to compare age between germinal and non-germinal centers. A $P$-value $<0.05$ was considered to be significant (GraphPad Prism software, version 3.02).

\section{Results}

All cases were stained for BCL6, CD10 and MUM1 and classified as germinal or non-germinal centers
Table 1 Overview of 50 diffuse large B-cell lymphoma cases

\begin{tabular}{|c|c|c|c|c|c|c|c|}
\hline & \multicolumn{2}{|c|}{ Total } & \multicolumn{2}{|c|}{$\begin{array}{l}\text { Germinal } \\
\text { center }\end{array}$} & \multicolumn{2}{|c|}{$\begin{array}{c}\text { Non-germinal } \\
\text { center }\end{array}$} & \multirow[t]{2}{*}{ P-value } \\
\hline & $\mathrm{n}=50$ & $\%$ & $\mathrm{n}=14$ & $\%$ & $\mathrm{n}=36$ & $\%$ & \\
\hline Age & & & & & & & $0.39^{\mathrm{a}}$ \\
\hline Median & 59 & & 55 & & 60 & & \\
\hline Range & 18-87 & & $27-81$ & & $18-87$ & & \\
\hline Gender & & & & & & & $0.91^{\mathrm{b}}$ \\
\hline Male & 31 & 63 & 9 & 64 & 20 & 56 & \\
\hline Female & 19 & 37 & 6 & 36 & 15 & 44 & \\
\hline Stage & & & & & & & $0.33^{\mathrm{b}}$ \\
\hline I & 35 & 71 & 8 & 53 & 28 & 72 & \\
\hline II & 15 & 29 & 6 & 47 & 8 & 28 & \\
\hline Localization & & & & & & & $0.15^{\mathrm{b}}$ \\
\hline Nodal & 19 & 37 & 8 & 53 & 11 & 31 & \\
\hline Extranodal & 31 & 63 & 6 & 47 & 25 & 69 & \\
\hline
\end{tabular}

\footnotetext{
a Age and location were tested using a Student's $t$-test.

${ }^{\mathrm{b}}$ Gender, stage and localization were tested using a $\chi^{2}$-test.
}

according to the algorithm described earlier by Hans et $a .^{3}$ Results for each case are given in Supplementary Table 2 . In total, $32 \%$ of the cases were positive for BCL6, $26 \%$ for CD10 and $52 \%$ for MUM1. The nodal diffuse large BCL group contained eight germinal and 11 non-germinal center cases. Eight central nervous system diffuse large BCL cases were classified as non-germinal and one as germinal center diffuse large BCL. For the testicular diffuse large BCL cases, ten cases were classified as non-germinal and one as germinal center. The eleven other extranodal cases were classified as germinal center in four cases and non-germinal center in seven cases. Comparison of the germinal center with the non-germinal center diffuse large BCL groups using a univariate analysis for age, gender, Ann Arbor stage and localization revealed no significant differences (Table 1).

In the total diffuse large BCL group, the expression levels of the individual miRNAs varied greatly (Figure 1). The most abundant miRNA, miR-21, showed a 100-fold higher expression level (median: 28), and miR-19b (median: 8.9) showed a 10-fold higher expression level than did miR-15a, miR-15b, miR-17-3p, miR-17-5p, miR-18a, miR-19a, miR-20a, miR-92a, miR-127, miR-155, miR-181a and miR-221. MiR-16 showed the lowest level of expression in comparison with the other miRNAs (median: 0.01; Table 2).

Within the germinal and non-germinal center groups, miR-21 showed the highest median expression level, 25 and 34, respectively (Table 2). The comparison of all 50 cases showed no significant differences between germinal and non-germinal center diffuse large BCLs for any of the 15 miRNAs. A second analysis was performed using only the nodal group of diffuse large BCL containing eight 
germinal and eleven non-germinal center cases, which again revealed no significant differences between the expression levels of the 15 miRNAs (Figure 2).

A separate analysis was made of the specific anatomical sites of presentation in testicular, central nervous system, nodal and other extranodal diffuse large BCL cases. MiR-17-5p showed a significantly higher expression level in central nervous system diffuse large BCLs in comparison with that in nodal and testicular diffuse large BCLs $(P=0.04$; Figure 3c). MiR-127 showed a significantly higher expression level in testicular diffuse large BCL in comparison with that in nodal and central nervous system diffuse large BCLs $(P<0.05$; Figure $3 \mathrm{~d})$. All other miRNAs showed no significant differences in these four diffuse large BCL subgroups.

We subsequently tested the cellular localization of the differentially expressed miRNAs using in situ

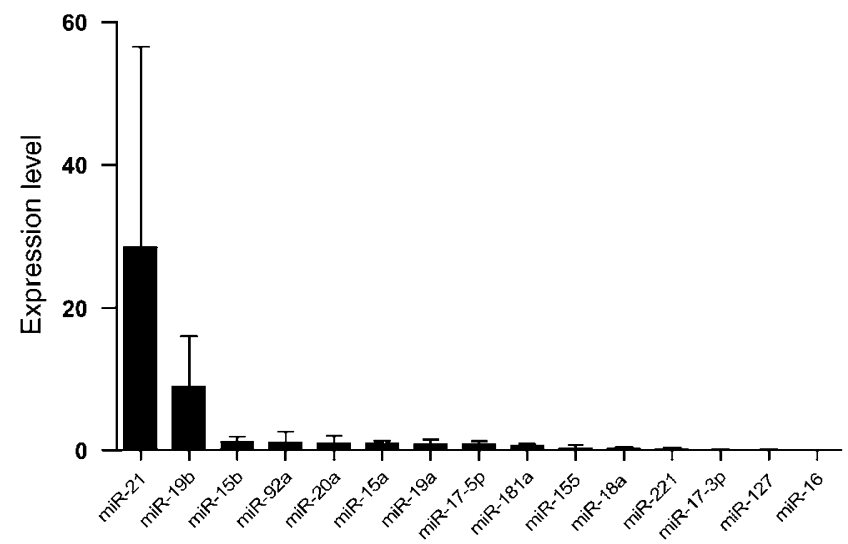

Figure 1 Expression levels of all 15 miRNAs. The median $(\mathrm{CI}=95 \%)$ expression levels of the 15 selected miRNAs show that miR-21 has a 100-fold higher expression level and miR-19b has a 10-fold higher expression level compared with the other 13 miRNAs. hybridization to confirm that the differential expression was related to higher expression levels in diffuse large BCL cells. RNA-ISH of miR-17-5p in all three central nervous system diffuse large BCL cases (Figure 3a), and that of miR-127 in all three testicular diffuse large BCL cases (Figure 3b) showed an almost restricted staining of tumor cells. Tumor cells showed cytoplasmic and nucleolar staining.

MiR-127 has only one proven target, ie, BCL6. Correlation between expression levels of miR-127 and expression levels of $B C L 6$ using qRT-PCR $\left(2^{-\Delta C_{\mathrm{t}}}\right)$ was significant $(P=0.027)$ with an inverse Spearman's coefficient $(r=-0.3)$ in the total diffuse large BCL group. We also compared the expression levels of miR-127 with the immunohistochemistry results for BCL6 protein expression. When the cases were divided into two groups on the basis of positive BCL6 staining $(n=15)$ and negative BCL6 staining $(n=25)$, expression levels of miR-127 were lower in the BCL6-positive (median level of 0.004) compared with that in BCL6-negative group (median: 0.07; $P=0.054)$.

\section{Discussion}

Diffuse large BCL is a lymphoma with a widely varying biological and clinical behavior and the different anatomical sites in which the presence of primary diffuse large BCL reflects these heterogeneous aspects. Earlier miRNA studies in diffuse large BCL usually comprised of a heterogeneous group containing cases with mixed sites of presentation. To determine the expression of B-cell-related miRNAs in diffuse large BCL and to examine the differences in miRNA levels in the germinal and non-germinal centers of different subgroups of diffuse large BCL and the relation to the site of presentation, we examined well-defined groups of primary nodal and extranodal diffuse large BCLs. To

Table 2 Median expression levels of all 15 miRNAs in diffuse large B-cell lymphoma subgroups

\begin{tabular}{|c|c|c|c|c|c|c|c|}
\hline & $\begin{array}{l}\text { All cases } \\
\mathrm{n}=50\end{array}$ & $\begin{array}{l}\text { Germinal } \\
\text { center } \\
\mathrm{n}=15\end{array}$ & $\begin{array}{l}\text { Non- } \\
\text { germinal } \\
\text { center } \\
\mathrm{n}=35\end{array}$ & $\begin{array}{l}\text { Nodal diffuse } \\
\text { large B-cell } \\
\text { lymphoma } \\
\mathrm{n}=19\end{array}$ & $\begin{array}{c}\text { Testicular diffuse } \\
\text { large B-cell } \\
\text { lymphoma } \\
\mathrm{n}=11\end{array}$ & $\begin{array}{c}\text { Central nervous } \\
\text { system diffuse large } \\
\text { B-cell lymphoma } \\
\mathrm{n}=9\end{array}$ & $\begin{array}{l}\text { Other } \\
\text { extranodal } \\
\mathrm{n}=11\end{array}$ \\
\hline miR-21 & 28 & 25 & 34 & 27 & 27 & 37 & 47 \\
\hline miR-19b & 8.9 & 9.6 & 8.7 & 9.4 & 9.7 & 8.6 & 7.3 \\
\hline miR-15b & 1.2 & 1.2 & 1.2 & 1.1 & 1.0 & 1.6 & 1.4 \\
\hline miR-92a & 1.0 & 0.9 & 1.1 & 0.8 & 2.2 & 1.3 & 1.0 \\
\hline miR-20a & 0.9 & 1.0 & 0.9 & 1.2 & 1.0 & 0.8 & 0.6 \\
\hline miR-15a & 0.9 & 1.0 & 0.9 & 0.9 & 1.5 & 0.9 & 1.0 \\
\hline miR-19a & 0.8 & 1.0 & 0.8 & 0.8 & 1.1 & 1.0 & 0.8 \\
\hline miR-17-5p & 0.8 & 0.7 & 0.9 & 0.6 & 1.1 & 1.6 & 0.6 \\
\hline miR-181a & 0.6 & 0.7 & 0.6 & 0.6 & 0.7 & 0.5 & 0.5 \\
\hline miR-155 & 0.3 & 0.2 & 0.3 & 0.3 & 0.3 & 0.6 & 0.1 \\
\hline miR-18a & 0.3 & 0.3 & 0.3 & 0.2 & 0.3 & 0.5 & 0.3 \\
\hline miR-221 & 0.2 & 0.2 & 0.2 & 0.2 & 0.1 & 0.3 & 0.2 \\
\hline miR-17-3p & 0.09 & 0.08 & 0.1 & 0.07 & 0.15 & 0.1 & 0.07 \\
\hline miR-127 & 0.03 & 0.04 & 0.03 & 0.02 & 0.14 & 0.02 & 0.02 \\
\hline miR-16 & 0.01 & 0.01 & 0.01 & 0.01 & 0.01 & 0.02 & 0.01 \\
\hline
\end{tabular}


a
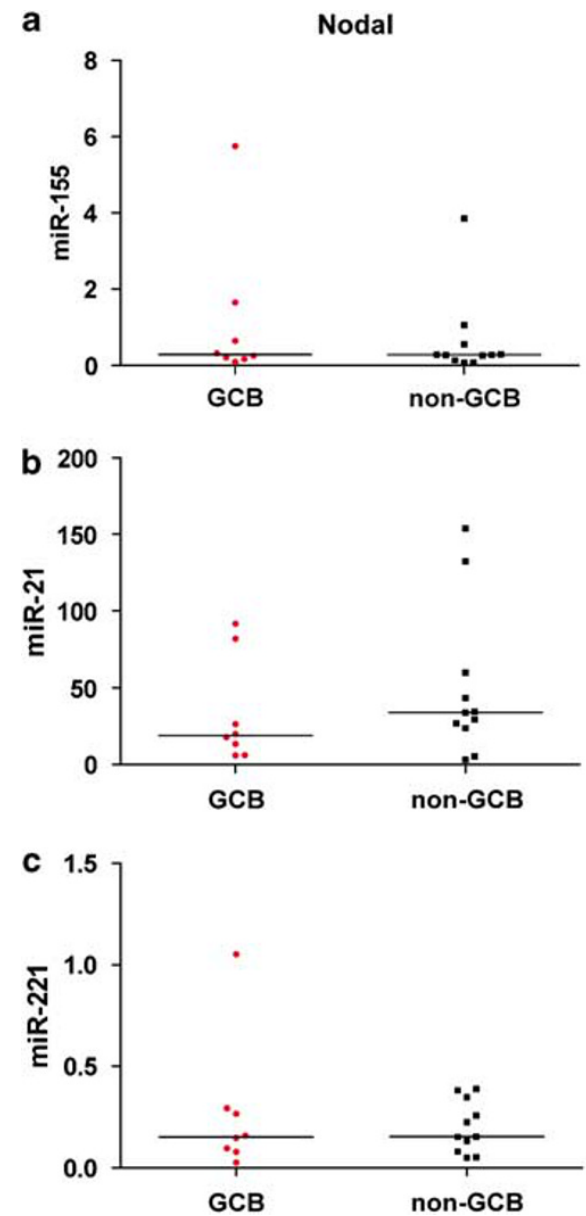
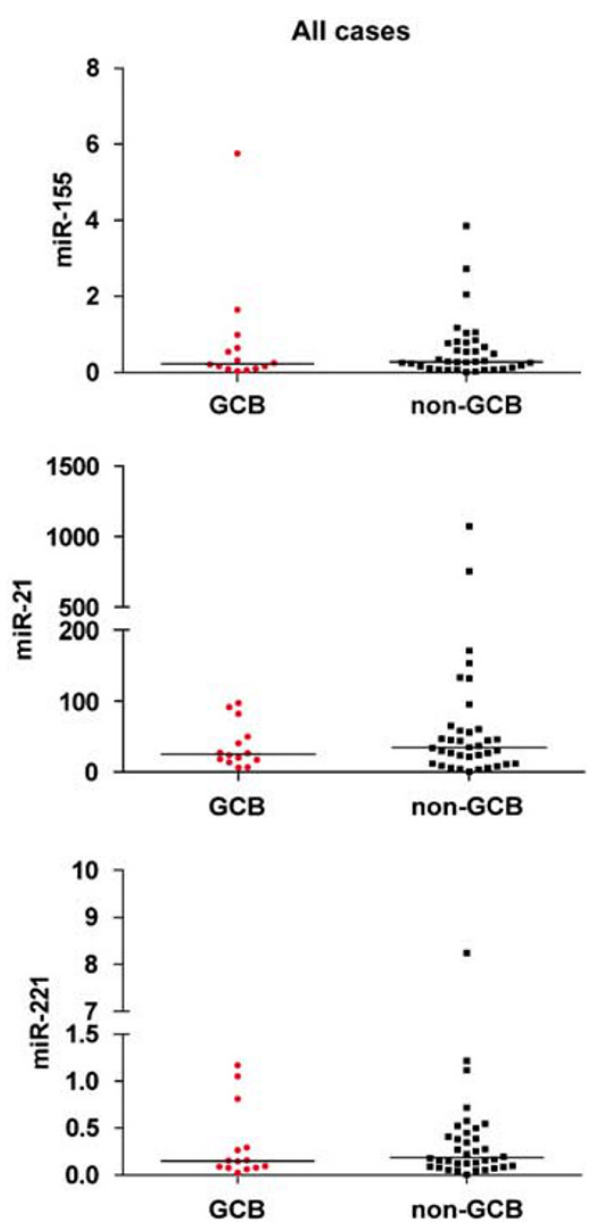

Figure 2 Similar expression levels of miR-155, miR-21 and miR-221 in nodal germinal and non-germinal center diffuse large B-cell lymphomas. The expression levels of miR-155 (a), miR-21 (b) and miR-221 (c) in nodal and in all 50 diffuse large B-cell lymphoma cases; the line indicates the median level. No significant differences are seen between the germinal and non-germinal center groups for any of the three miRNAs either only for the nodal cases or for the total group of diffuse large B-cell lymphoma cases.

that end, we selected only Ann Arbor stage I and II cases with nodal, central nervous system or testicular presentation, as well as a heterogeneous group of primary extranodal diffuse large BCL, also only stage I or II. We used paraffin-embedded tissue for our analysis to allow the inclusion of a larger number of cases compared with that in frozen samples. On the basis of recent publications, it is evident that miRNA expression studies result in comparable results for both paraffin and frozen specimens. ${ }^{21,28}$

Consistent with the literature, we found that the testicular diffuse large BCL and central nervous system diffuse large BCL were predominantly nongerminal center. ${ }^{29,30}$ The distribution in the nodal and the heterogeneous other extranodal diffuse large BCL groups was also consistent with earlier reports. ${ }^{2,4}$ Remarkably, no significant differences in expression levels for any of the 15 B-cell related miRNAs were found between the germinal and nongerminal center cases in our total group of diffuse large BCL. In earlier reports, miR-155, miR-21 and miR-221 levels were shown to be higher in non-germinal center diffuse large BCL. ${ }^{18,20,21}$ A possible explanation for these differences might be that we used specific localizations, ie, primary nodal, testicular, central nervous system and a group of heterogeneous extranodal diffuse large BCL. Earlier reports have used groups of diffuse large BCL with mixed locations and varying Ann Arbor stages. Eis et $a l^{20}$ compared a heterogeneous group with respect to presentation and stage containing only four germinal center and nineteen activated diffuse large BCLs. Kluiver et $a l^{18}$ investigated a group of eighteen diffuse large BCLs from which eleven were extranodal, presenting at eight different anatomical sites. Lawrie et $a l^{21}$ used a heterogeneous group of 35 de novo diffuse large BCLs from which 23 cases presented with stage III or IV disease, making a distinction between primary nodal and extranodal almost impossible. Lack of differential expression of these three miRNAs between germinal and non-germinal center diffuse large BCLs in our study cannot be attributed to the inclusion of central nervous system and testicular cases, as analysis of the stage I and II nodal groups 
and the primary stage I and II heterogeneous extranodal groups also showed no differences for miR-155, miR-21 and miR-221, or for the other 12
miRNAs. This suggests that the differences observed in earlier studies and the lack of differential expression in our stage I and II diffuse large BCL
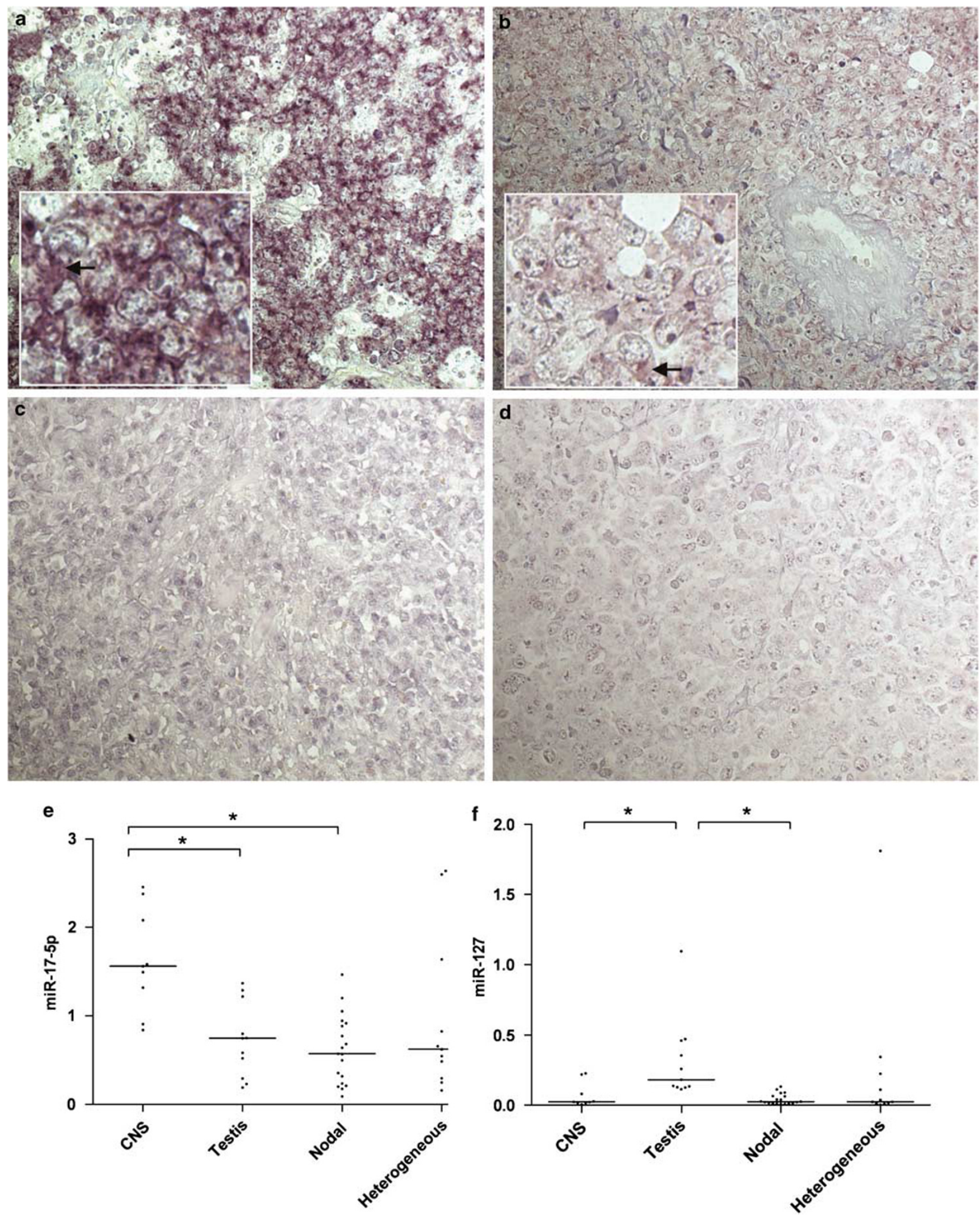
cases may be related to the inclusion of heterogeneous diffuse large BCL cases.

Using qRT-PCR, we found two highly expressed miRNAs, miR-21 and miR-19b, in the diffuse large BCL group. The most abundant, miR-21, showed levels that were 10-fold higher than that in miR-19b and 100-fold higher than that in any of the other 13 miRNAs. Our data are consistent with Lawrie et $a l^{21}$ who also showed high miR-21 levels in diffuse large BCL. Interestingly, miR-21 has been shown to be highly expressed in a variety of different tumors and is associated with the downregulation of BCL2 ${ }^{31}$ and PTEN. ${ }^{32}$ MiR-19b also showed a high expression with a 10-fold higher expression level than the other 13 miRNAs. MiR-19b is one of the seven miRNAs in the polycistron, C13ORF25. C13ORF25 is overexpressed in $70 \%$ of diffuse large BCLs with 13q31q32 amplification. Although the miR-17-92 has two homologous clusters, the miR-106a-92 cluster on chromosome $\mathrm{X}$ and the miR-106b-25 cluster on chromosome 7 , it is the amplification of the $13 q 31$ locus that has been linked to the overexpression of miR-17-92. ${ }^{24,33}$

MiR-17-5p, another member of the C13ORF25 polycistron, was differentially expressed between the subgroups of diffuse large BCL, showing a significantly higher expression level in central nervous system diffuse large BCL. C13ORF25 was recently shown to be upregulated by binding of MYC to its promoter region. ${ }^{34}$ Mice models have shown that the overexpression of miR-17-92 reduces apoptosis, and further studies have shown that miR-17-5p and miR20 target the proapoptotic E2F1 gene. ${ }^{35}$ Differences in the levels of miR-19b and miR-17-5p when compared with those in the five other miRNAs in the C13ORF25 cluster may indicate variation in processing efficiency or stability between the nodal, testicular and central nervous system diffuse large BCL subgroups. Interestingly, central nervous system diffuse large BCL has been shown to have a higher expression of MYC than the nodal diffuse large BCL. ${ }^{36}$ In central nervous system diffuse large BCL, only the miR-17-5p and miR-20a cluster members were expressed at a significantly higher level, especially in comparison with that in the nodal group. This indicates that both MYC and differences in processing and stability of the mature miRNAs of C13ORF25 may contribute to the higher expression levels of 17-5p in central nervous system diffuse large BCL.

In the testicular diffuse large BCL, miR-127 levels were significantly higher compared with that in the nodal and central nervous system diffuse large BCLs. Earlier studies have shown that the expression of miR-127 was upregulated after treatment with chromatin-modifying drugs, and this induced the downregulation of the proto-oncogene BCL6. Transfection of miR-127, precursors in Ramos cells, designed to mimic endogenous miR-127 caused the downregulation of BCL6 protein. ${ }^{10}$ We showed a negative correlation between the expression of BCL6 and the expression for miR-127 in diffuse large BCL, which supports the earlier finding that BCL6 is a target for miR-127. BCL6 transcription factor is expressed in germinal centers and is associated with germinal center events. ${ }^{37}$ It is one of the major transcriptional regulators of centroblasts and inhibits the differentiation of GC B cells into plasma and memory cells. ${ }^{38}$ In germinal center diffuse large BCL, low miR-127 levels can result in high BCL6 expression levels, and thereby keep the tumor cells in the GC developmental stage, giving a proliferative advantage. The high level of miR-127 in testicular diffuse large BCL can therefore induce the downregulation of BCL6, which is consistent with the non-germinal center phenotype of testicular diffuse large BCL.

Differential expression of specific miRNAs in testicular and central nervous system lymphomas could also be caused by expression in preexistent non-malignant cells, such as Sertoli cells or astrocytes, which might be present in the tumor tissues. We have excluded this possibility by selecting cases with $>80 \%$ tumor cells and by the application of in situ hybridization for the two relevant miRNAs, which revealed a selective expression in tumor cells. Besides cytoplasmic staining, we also observed nucleolar staining. This may be caused by non-specific binding of the probe, although recent reports have suggested mechanisms for miRNA localization in the nucleus ${ }^{39}$ and nucleolus. ${ }^{40}$ Furthermore, miRNA expression studies on a wide range of normal tissues, including normal brain and testis, using qRT-PCR, showed that the miR-127 and miR-17-5p expression levels are contrary to those found in testicular and central nervous system diffuse large BCLs. ${ }^{41}$

The heterogeneous biology of diffuse large BCL implies that it is essential to select homogeneous groups of diffuse large BCL for pathogenetic studies. In our study, we used strict subsets of diffuse large BCL that were proven to have been derived from three specific sites. By showing significantly different expression levels for miR-17-5p and miR-127 in

Figure 3 Significant differences in the expression levels for miR-17-5p and miR-127. (a) In situ hybridization for miR-17-5p shows cytoplasmic and nucleolar staining not only in tumor cells of central nervous system diffuse large B-cell lymphoma but also in cells that do not stain for miR-17-5p. (b) The in situ hybridization for miR-127 also shows specific cytoplasmatic and nucleolar staining in tumor cells of testicular diffuse large B-cell lymphoma, but not in the Sertoli cells. (c) A negative DLBCL case that showed no detectable staining for miR-17-5p. (d) A negative DLBCL case that showed no detectable staining for miR-127. (e) MiR-17-5p shows a significant difference in median expression levels between the diffuse large B-cell lymphoma groups, with the highest expression in central nervous system diffuse large B-cell lymphoma. (f) MiR-127 shows a higher expression level, specifically for testicular diffuse large B-cell lymphoma. $\left({ }^{*} P<0.05\right)$. 
central nervous system diffuse large BCL and in testicular diffuse large BCL, we support the assumption that each location appears to be a separate biological entity.

\section{References}

1 Colomo L, Loong F, Rives S, et al. Diffuse large B-cell lymphomas with plasmablastic differentiation represent a heterogeneous group of disease entities. Am J Surg Pathol 2004;28:736-747.

2 De PP, De Wolf-Peeters C. Diffuse large B-cell lymphoma: a heterogeneous group of non-Hodgkin lymphomas comprising several distinct clinicopathological entities. Leukemia 2007;21:37-43.

3 Hans CP, Weisenburger DD, Greiner TC, et al. Confirmation of the molecular classification of diffuse large B-cell lymphoma by immunohistochemistry using a tissue microarray. Blood 2004;103:275-282.

4 Alizadeh AA, Eisen MB, Davis RE, et al. Distinct types of diffuse large B-cell lymphoma identified by gene expression profiling. Nature 2000;403:503-511.

5 Lossos IS, Czerwinski DK, Alizadeh AA, et al. Prediction of survival in diffuse large-B-cell lymphoma based on the expression of six genes. N Engl J Med 2004;350: 1828-1837.

6 Coiffier B, Lepage E, Briere J, et al. CHOP chemotherapy plus rituximab compared with CHOP alone in elderly patients with diffuse large-B-cell lymphoma. N Engl J Med 2002;346:235-242.

7 Barrans SL, Carter I, Owen RG, et al. Germinal center phenotype and bcl-2 expression combined with the International Prognostic Index improves patient risk stratification in diffuse large B-cell lymphoma. Blood 2002;99:1136-1143.

8 Wilson KS, Sehn LH, Berry B, et al. CHOP-R therapy overcomes the adverse prognostic influence of BCL-2 expression in diffuse large B-cell lymphoma. Leuk Lymphoma 2007;48:1102-1109.

9 Bartel DP. MicroRNAs: genomics, biogenesis, mechanism, and function. Cell 2004;116:281-297.

$10 \mathrm{Lu} \mathrm{J}$, Getz G, Miska EA, et al. MicroRNA expression profiles classify human cancers. Nature 2005;435: 834-838.

11 Calin GA, Croce CM. MicroRNA signatures in human cancers. Nat Rev Cancer 2006;6:857-866.

12 Lawrie CH. MicroRNAs and haematology: small molecules, big function. Br J Haematol 2007;137:503-512.

13 Jay C, Nemunaitis J, Chen P, et al. miRNA profiling for diagnosis and prognosis of human cancer. DNA Cell Biol 2007;26:293-300.

14 Calin GA, Liu CG, Sevignani C, et al. MicroRNA profiling reveals distinct signatures in B cell chronic lymphocytic leukemias. Proc Natl Acad Sci USA 2004;101:11755-11760.

15 Kluiver J, Kroesen BJ, Poppema S, et al. The role of microRNAs in normal hematopoiesis and hematopoietic malignancies. Leukemia 2006;20:1931-1936.

16 Chen CZ, Lodish HF. MicroRNAs as regulators of mammalian hematopoiesis. Semin Immunol 2005;17: 155-165.

17 Chen CZ, Li L, Lodish HF, et al. MicroRNAs modulate hematopoietic lineage differentiation. Science 2004; 303:83-86.
18 Kluiver J, Poppema S, de JD, et al. BIC and miR-155 are highly expressed in Hodgkin, primary mediastinal and diffuse large B cell lymphomas. J Pathol 2005;207: 243-249.

19 Cimmino A, Calin GA, Fabbri M, et al. miR-15 and miR-16 induce apoptosis by targeting BCL2. Proc Natl Acad Sci USA 2005;102:13944-13949.

20 Eis PS, Tam W, Sun L, et al. Accumulation of miR-155 and BIC RNA in human B cell lymphomas. Proc Natl Acad Sci USA 2005;102:3627-3632.

21 Lawrie CH, Soneji S, Marafioti T, et al. Microrna expression distinguishes between germinal center $\mathrm{B}$ cell-like and activated B cell-like subtypes of diffuse large B cell lymphoma. Int J Cancer 2007;121:1156-1161.

22 Costinean S, Zanesi N, Pekarsky Y, et al. Pre-B cell proliferation and lymphoblastic leukemia/high-grade lymphoma in E(mu)-miR155 transgenic mice. Proc Natl Acad Sci USA 2006;103:7024-7029.

23 Rao PH, Houldsworth J, Dyomina K, et al. Chromosomal and gene amplification in diffuse large B-cell lymphoma. Blood 1998;92:234-240.

24 Ota A, Tagawa H, Karnan S, et al. Identification and characterization of a novel gene, C13orf25, as a target for 13q31-q32 amplification in malignant lymphoma. Cancer Res 2004;64:3087-3095.

25 Tagawa H, Seto M. A microRNA cluster as a target of genomic amplification in malignant lymphoma. Leukemia 2005;19:2013-2016.

26 van den Berg A, Kroesen BJ, Kooistra K, et al. High expression of B-cell receptor inducible gene BIC in all subtypes of Hodgkin lymphoma. Genes Chromosomes Cancer 2003;37:20-28.

27 Specht K, Richter T, Muller U, et al. Quantitative gene expression analysis in microdissected archival formalin-fixed and paraffin-embedded tumor tissue. Am J Pathol 2001;158:419-429.

28 Xi Y, Nakajima G, Gavin E, et al. Systematic analysis of microRNA expression of RNA extracted from fresh frozen and formalin-fixed paraffin-embedded samples. RNA 2007;13:1668-1674.

29 Booman M, Douwes J, Glas AM, et al. Primary testicular diffuse large B-cell lymphomas have activated B-cell-like subtype characteristics. J Pathol 2006;210: 163-171.

30 Levy O, Deangelis LM, Filippa DA, et al. Bcl-6 predicts improved prognosis in primary central nervous system lymphoma. Cancer 2008;112:151-156.

31 Si ML, Zhu S, Wu H, et al. miR-21-mediated tumor growth. Oncogene 2007;26:2799-2803.

32 Meng F, Henson R, Wehbe-Janek H, et al. MicroRNA-21 regulates expression of the PTEN tumor suppressor gene in human hepatocellular cancer. Gastroenterology 2007;133:647-658.

33 Migliazza A, Bosch F, Komatsu H, et al. Nucleotide sequence, transcription map, and mutation analysis of the 13q14 chromosomal region deleted in B-cell chronic lymphocytic leukemia. Blood 2001;97:2098-2104.

34 Rinaldi A, Poretti G, Kwee I, et al. Concomitant MYC and microRNA cluster miR-17-92 (C13orf25) amplification in human mantle cell lymphoma. Leuk Lymphoma 2007;48:410-412.

35 O'Donnell KA, Wentzel EA, Zeller KI, et al. c-Mycregulated microRNAs modulate E2F1 expression. Nature 2005;435:839-843.

36 Rubenstein JL, Fridlyand J, Shen A, et al. Gene expression and angiotropism in primary CNS lymphoma. Blood 2006;107:3716-3723. 
37 Cattoretti G, Chang CC, Cechova K, et al. BCL-6 protein is expressed in germinal-center B cells. Blood 1995; $86: 45-53$.

38 Shaffer AL, Yu X, He Y, et al. BCL-6 represses genes that function in lymphocyte differentiation, inflammation, and cell cycle control. Immunity 2000;13:199-212.

39 Politz JC, Zhang F, Pederson T. MicroRNA-206 colocalizes with ribosome-rich regions in both the nucleolus and cytoplasm of rat myogenic cells. Proc Natl Acad 555 Sci USA 2006;103:18957-18962.

40 Hwang HW, Wentzel EA, Mendell JT. A hexanucleotide element directs microRNA nuclear import. Science 2007;315:97-100.

41 Liang Y, Ridzon D, Wong L, et al. Characterization of microRNA expression profiles in normal human tissues. BMC Genomics 2007;8:166.

Supplementary Information accompanies the paper on Modern Pathology website (http://www.nature.com/ modpathol) 